\title{
Quantum dot emission from site-controlled InGaN/GaN micropyramid arrays
}

\author{
P. R. Edwards ${ }^{\text {a) }}$ and R. W. Martin \\ Department of Physics, University of Strathclyde, 107 Rottenrow, Glasgow G4 ONG, United Kingdom \\ I. M. Watson and C. Liu \\ Institute of Photonics, University of Strathclyde, 106 Rottenrow, Glasgow G4 ONW, United Kingdom \\ R. A. Taylor, J. H. Rice, J. H. Na, J. W. Robinson, and J. D. Smith \\ Clarendon Laboratory, University of Oxford, Parks Road, Oxford OX1 3PU, United Kingdom
}

(Received 25 May 2004; accepted 9 September 2004)

\begin{abstract}
$\mathrm{In}_{x} \mathrm{Ga}_{1-x} \mathrm{~N}$ quantum dots have been fabricated by the selective growth of $\mathrm{GaN}$ micropyramid arrays topped with InGaN/GaN quantum wells. The spatially, spectrally, and time-resolved emission properties of these structures were measured using cathodoluminescence hyperspectral imaging and low-temperature microphotoluminescence spectroscopy. The presence of InGaN quantum dots was confirmed directly by the observation of sharp peaks in the emission spectrum at the pyramid apices. These luminescence peaks exhibit decay lifetimes of approximately $0.5 \mathrm{~ns}$, with linewidths down to $650 \mu \mathrm{eV}$ (limited by the spectrometer resolution). (C) 2004 American Institute of Physics.
\end{abstract}

[DOI: $10.1063 / 1.1815043$ ]

Semiconductor quantum dots (QDs) confine carriers in all three spatial dimensions, resulting in discrete energy spectra analogous to those of atoms. A number of III-nitridebased QD nanostructure systems (e.g., InGaN and $\mathrm{GaN}$ ) have been demonstrated. ${ }^{1-3}$ These materials possess interesting electronic properties, such as strong piezoelectric fields and high exciton binding energies, that are not found in the widely studied arsenide system.

Most III-nitride-based QD nanostructure systems reported to date have consisted of QDs that are distributed randomly across the sample area. This is a consequence of using the Stranski-Krastanov (SK) growth method, involving self-assembly of the QDs on a previously grown wetting layer. Some applications by contrast require the precise positioning of QDs on a substrate, for which an alternative to SK growth is required. Arrays of site-controlled InGaAs QDs have been fabricated, however, through the use of lithography to define the region where the growth of the QDs will occur. ${ }^{4}$ Previous studies involving the selective growth of $\mathrm{InGaN} / \mathrm{GaN}$ nanostructures have reported the presence of QDs as evidenced by a blueshift in the emission ${ }^{5}$ and by the small size of the emitting region, ${ }^{6}$ although no sharp-line QD luminescence was observed.

In this letter, we report on the growth and high spatial resolution spectroscopy of arrays of site-controlled InGaN/GaN QDs positioned at the apices of GaN micropyramids. The pyramids were formed by metalorganic chemical vapor deposition of $\mathrm{GaN}$ through an array of circular holes lithographically patterned into a silica mask on a GaNon-sapphire substrate. The crystal symmetry of the wurtzite $\mathrm{GaN}$ results in the formation of hexagonal-based pyramidal structures, with facets formed by $\{10 \overline{1} 1\}$ planes. ${ }^{7}$ The structures were $\sim 8 \mu \mathrm{m}$ in height, with base edges of $\sim 5 \mu \mathrm{m}$, and were repeated in a hexagonal array with a $10 \mu \mathrm{m}$ pitch to give an areal density of $2 \times 10^{6} \mathrm{~cm}^{-2}$. A 5-period $\mathrm{In}_{x} \mathrm{Ga}_{1-x} \mathrm{~N}$ quantum well (QW) structure was subsequently

${ }^{a)}$ Electronic mail: paul.edwards@strath.ac.uk grown on the top surface in order to form QDs at the sharp apices. The QWs were grown using conditions calibrated for nonselective planar growth, for which InGaN wells of $2.0 \mathrm{~nm}$ and $\mathrm{GaN}$ barriers of $6.0 \mathrm{~nm}$ gave a structure emitting at $2.75 \mathrm{eV}$.

Cathodoluminescence (CL) hyperspectral imaging was carried out in a modified Cameca SX100 electron probe microanalyzer, in which emitted light is collected using an optical microscope that is coaxial and confocal with the electron beam. ${ }^{8}$ The sample position was scanned in $100 \mathrm{~nm}$ steps with respect to the stationary beam, with the roomtemperature CL spectrum recorded at each position using a $0.125 \mathrm{~m}$ spectrometer and cooled charge coupled device (CCD) array. A $500 \mathrm{pA}$ beam with an accelerating voltage of $5 \mathrm{kV}$ was used, with a detection time of $200 \mathrm{~ms}$ per pixel.

Spatially resolved time-integrated photoluminescence (PL) measurements and time-resolved single-photon counting PL measurements were made using a frequency-tripled Ti:Sapphire laser at $266 \mathrm{~nm}$ (100 fs pulse width) in a temperature-controlled microscope cryostat. The laser was focused through a $36 \times$ microscope objective lens to a spot size of $\sim 2 \mu \mathrm{m}$, which was aligned on the sample surface using a CCD camera. This allowed the laser spot to be focused on the pyramid apex, on the side wall, or in the space between pyramids. The PL was collected by the same microscope objective lens. Time-integrated PL spectra were recorded using a cooled CCD camera mounted on a $0.3 \mathrm{~m}$ monochromator, which had a 1200 grooves $/ \mathrm{mm}$ grating. Time-resolved PL measurements were performed using a second identical monochromator, but now using a timecorrelated single-photon counting detection system based on a fast photomultiplier tube (PMT). The PMT used gave a time resolution of $150 \mathrm{ps}$. Once a single QD had been identified using the CCD system, the luminescence could be switched to the PMT system enabling a time-resolved trace from an identified QD to be recorded.

Figures 1 and 2 show subsets of a single roomtemperature CL hyperspectral image of a micropyramid. Figure 1(a) shows a spectrum from the center of one of the 


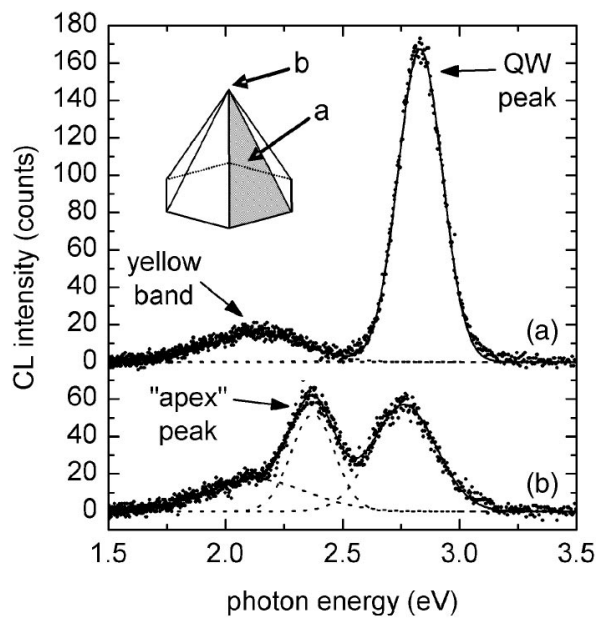

FIG. 1. Representative room-temperature CL spectra extracted from points on the hyperspectral image, showing the emission from (a) a facet center and (b) the apex. The lines show the results of fitting three Gaussian peaks to each spectrum, a process repeated for each pixel position in the CL dataset.

facets, exhibiting strong QW luminescence at $\sim 2.8 \mathrm{eV}$ together with a weaker "yellow band" centered at $\sim 2.2 \mathrm{eV}$. Figure 1(b) shows (on the same scale) the spectrum at the apex, in which an additional peak at $2.37 \mathrm{eV}$ is present. Fitting a Gaussian to each of these peaks using nonlinear regression, and repeating for each pixel position in the scan, allows low-noise images to be assembled representing the spatial dependence of these spectral features. Examples of these are shown in Fig. 2. Figures 2(a) and 2(c) show the intensity and peak energy of the QW peak, respectively. This luminescence is seen to emanate from the entire pyramidal surface with the exception of the apex, at which position it is less intense. (The symmetrical "halo" effect of increased intensity on the facets midway between the base and apex may be attributable to waveguiding effects within the $\mathrm{GaN}$
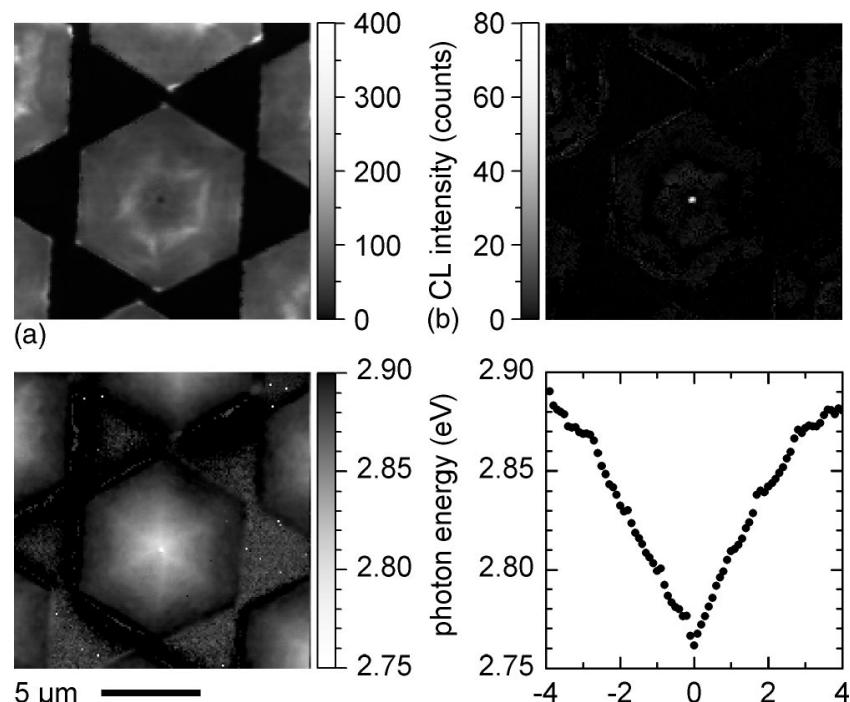

(c)

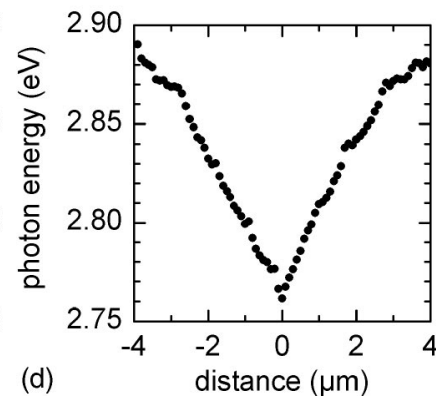

FIG. 2. Images constructed from parameters determined by fitting three Gaussian peaks to each of the $150 \times 150$ spectra in the CL hyperspectral image. (a) and (c) show the peak intensity and energy respectively of the QW emission from the facets, while (b) shows the intensity of the $2.37 \mathrm{eV}$ apex-related band. The line scan (d), showing the QW peak energy varying across the pyramid, was extracted from a horizontal line through the apex

in (c). $\quad 650 \mu \mathrm{eV}$ (a value limited by the resolution of the spectrom-
Downloaded 09 Nov 2004 to 130.159.248.44. Redistribution subject to AlP license or copyright, see http://apl.aip.org/apl/copyright.jsp
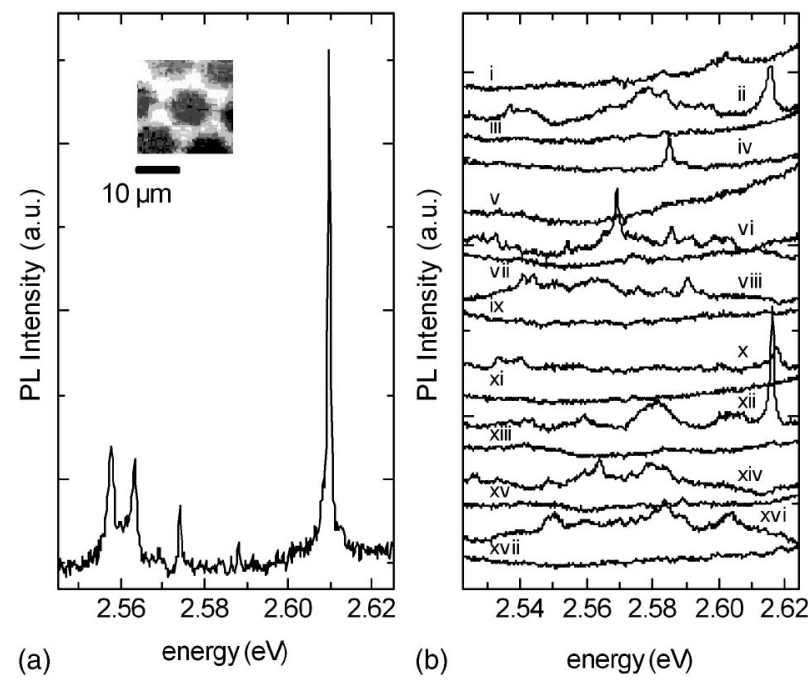

FIG. 3. Spatially resolved PL spectra obtained at $4.2 \mathrm{~K}$. The spectrum recorded from the apex of a single pyramid (a) exhibits several very sharp lines corresponding to optical transitions from exciton complexes from up to five QDs. An optical image of the sample surface, showing the hexagonal pyramids, is shown in the inset. A series of spectra (b) were recorded in $5 \mu \mathrm{m}$ steps across the sample surface: i, iii, v, vii, ix, xi, xiii, xv, and xvii are spectra recorded at positions between the apices of each pyramid; whereas ii, iv, vi, viii, x, xii, xiv, and xvi show spectra recorded at the apices.

pyramid. $)^{9}$ The emission energy of this peak is found to vary significantly [Fig. 2(c)], decreasing with proximity to the apex. This effect is seen more clearly in a linescan through the centre of the pyramid, shown in Fig. 2(d), in which the shift appears linear with distance from the apex.

Relaxation of compressive strain with increasing growth thickness may be expected to contribute to the observed redshift in such structures, ${ }^{10}$ but such an effect would not be expected to persist to the apex (a height of $\sim 8 \mu \mathrm{m}$ ). The effect is however consistent with temperature gradients during QW growth: The InN fraction $x$ in $\operatorname{In}_{x} \mathrm{Ga}_{1-x} \mathrm{~N}$ increases strongly with decreasing growth temperature, resulting in more indium being incorporated at the topographic extremes where radiative thermal losses are higher.

Figure 2(b) shows the intensity of the $2.37 \mathrm{eV}$ peak [as seen in Fig. 1(b)], emitted only from the pyramid apex. This observation is similar to that made by Tachibana et al. ${ }^{6}$ at room temperature on comparable structures (allowing for a peak shift due to a different $\operatorname{In}_{x} \mathrm{Ga}_{1-x} \mathrm{~N}$ composition), which was interpreted by them as evidence of QD formation. In order to distinguish more confidently between genuine QD emission and other possible luminescence sources (e.g., defects), low-temperature spatially resolved luminescence measurements are required.

Figure 3(a) shows the spatially resolved PL spectrum obtained from the tip of a single pyramidal structure at 4.2 K. A white-light contrast image taken with the objective lens used to excite the sample is shown in the inset, clearly showing the outline of the hexagonal pyramids. The PL spectrum from the pyramid apex reveals sharp peaks characteristic of localized states observed in PL from single InGaN QDs. ${ }^{3,11}$ The spectrum exhibits one strong peak and four weaker peaks; it is unclear whether the weaker peaks are due to higher-order processes or exciton transitions in the other QDs expected to be present at the pyramid tip. The full width at half maximum of the peaks was found to be as small as $650 \mu \mathrm{eV}$ (a value limited by the resolution of the spectrom- 


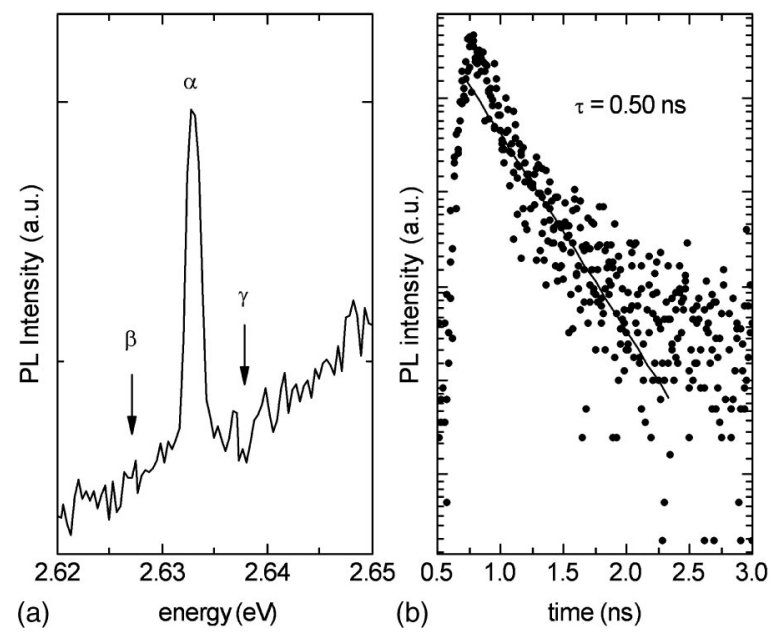

FIG. 4. Spatially resolved PL from a single QD at $4.2 \mathrm{~K}$. The spectrum (a) shows a sharp peak originating from an optical transition in a single QD. The decay trace (b) for this peak was separated from the background QW emission by recording traces at $\beta$ and $\gamma$ and subtracting the average of these traces from that recorded at peak $\alpha$.

eter). Figure 3(b) shows a series of PL spectra recorded over the sample surface. The laser spot was sequentially focused at adjacent pyramid apices and at points midway between apices. Sharp peaks were observed at the centers of each of the pyramids (ii, iv, vi, etc.) and not elsewhere on the sample (i, iii...). These observations support the assertion that QDs are present at the pyramid apices where the facet QW planes intersect.

It should be noted that these sharp peaks do not appear in the same spectral region as the broad apex-related CL peak seen in Figs. 1(b) and 2(b), even after allowing for the difference in temperature. Moreover, the intensities of the sharp QD peaks were found to diminish rapidly with increasing temperature, becoming undetectable above $\sim 90 \mathrm{~K}$. We therefore conclude that the luminescence emitted from the apex region at room temperature does not itself indicate the presence of QDs (as was previously reported), ${ }^{6}$ but may instead be defect related. The redshifting of the sharp QD peaks with respect to the QW luminescence is likely to result from larger critical dimensions and higher built-in electric fields, as discussed in earlier work. ${ }^{8}$

Figure 4(a) shows a micro-PL spectrum of a single InGaN QD, showing a sharp peak (marked $\alpha$ ) superimposed upon a strong background. This background originates from excitation of the side QW. It is therefore expected that the PL decay trace recorded for the single InGaN QD will possess a dynamic profile with contributions from both the side well and the QD. Since the single QD emission is sharp, monitoring the PL $1 \mathrm{~nm}$ on each side of the wavelength corresponding to that of the InGaN QD [at $\beta$ and $\gamma$ shown in Fig. 4(a)] produces PL exclusively from the side well. In this way, it is possible to use the time-resolved traces on each side of the QD emission to correct for the well emission collected at the same spectral position as the QD emission. Subtraction of this background enables the decay profile from the single InGaN QD to be determined. This is shown in Fig. 4(b). The PL decay trace from the side $\mathrm{QW}$ is nonexponential, in agreement with previous measurements, ${ }^{12}$ and exhibits a fast initial decay followed by a slower component. The trace from the QD closely matches that observed from the side well in both shape and lifetime. The decay time was parameterized by fitting a straight line to a portion of the decay trace, plotted on a logarithmic scale, and was found to be $\sim 0.50 \mathrm{~ns}$, which is much shorter than the lifetimes of $\sim 2 \mathrm{~ns}$ reported for InGaN QDs grown by self-assembly techniques. ${ }^{13}$ The shorter exciton recombination time in selectively grown QDs is believed to be due to the stronger coupling of the zero-dimensional QD state to the side QWs in this system, in contrast to self-assembled nanostructures where the QD is relatively decoupled from an underlying wetting layer.

In summary, we have grown site-controlled arrays of QDs by the deposition of InGaN/GaN QWs on GaN micropyramids. The zero-dimensional nature of these structures has been directly confirmed by the observation of narrow $(\sim 650 \mu \mathrm{eV})$ emission peaks emitted from the pyramid tips. This QD luminescence has a decay lifetime of $\sim 0.50 \mathrm{~ns}$, and has been distinguished from a suspected defect band also emitted from the vicinity of the apices.

The authors thank H. M. H. Chong and R. M. De La Rue of the University of Glasgow for the lithographic patterning. This work was supported by EPSRC.

${ }^{1}$ F. Widmann, B. Daudin, G. Feuillet, Y. Samson, J. L. Rouvière, and N. Pelekanos, J. Appl. Phys. 83, 7618 (1998).

${ }^{2}$ K. Tachibana, T. Someya, and Y. Arakawa, Appl. Phys. Lett. 74, 383 (1999).

${ }^{3}$ R. A. Oliver, G. A. D. Briggs, M. J. Kappers, C. J. Humphreys, J. H. Rice, J. D. Smith, and R. A. Taylor, Appl. Phys. Lett. 83, 755 (2003).

${ }^{4}$ M. H. Baier, E. Pelucchi, E. Kapon, S. Varoutsis, M. Gallart, I. RobertPhilip, and I. Abram, Appl. Phys. Lett. 84, 648 (2004).

${ }^{5}$ J. Wang, M. Nozaki, M. Lachab, Y. Ishikawa, R. S. Qhalid Fareed, T. Wang, M. Hao, and S. Sakai, Appl. Phys. Lett. 75, 950 (1999).

${ }^{6}$ K. Tachibana, T. Someya, S. Ishida, and Y. Arakawa, Appl. Phys. Lett. 76, $3212(2000)$.

${ }^{7}$ S. Kitamura, K. Hiramatsu, and N. Sawaki, Jpn. J. Appl. Phys., Part 2 34, L1184 (1995).

${ }^{8}$ R. W. Martin, P. R. Edwards, K. P. O’Donnell, M. D. Dawson, C.-W. Jeon, C. Liu, G. R. Rice, and I. M. Watson, Phys. Status Solidi A 201, 665 (2004).

${ }^{9}$ H. X. Jiang, J. Y. Lin, K. C. Zeng, and W. Yang, Appl. Phys. Lett. 75, 763 (1999).

${ }^{10}$ F. Bertram, J. Christen, M. Schmidt, K. Hiramatsu, S. Kitmura, and N. Sawaki, Physica E (Amsterdam) 2, 552 (1998).

${ }^{11}$ O. Moriwaki, T. Someya, K. Tachibana, S. Ishida, and Y. Arakawa, Appl. Phys. Lett. 76, 2361 (2000).

${ }^{12}$ I. L. Krestnikov, N. N. Ledentsov, A. Hoffmann, D. Bimberg, A. V. Sakharov, W. V. Lundin, A. F. Tsatsul'nikov, A. S. Usikov, Z. I. Alferov, Y. G. Musikhin, and D. Gerthsen, Phys. Rev. B 66, 155310 (2002).

${ }^{13}$ J. Y. Robinson, J. H. Rice, A. Jarjour, J. D. Smith, R. A. Taylor, R. A. Oliver, G. A. D. Briggs, M. J. Kappers, C. J. Humphreys, and Y. Arakawa, Appl. Phys. Lett. 83, 2674 (2003). 